\title{
Excited state intramolecular proton transfer fluorescence emission of $o$-hydroxyphenyl-triazine derivatives
}

\author{
LI HaiLi, LI HongRu*, YANG Long, XU XiaoFang, ZHANG ShengTao \& GAO Fang* \\ College of Chemistry and Chemical Engineering, Chongqing University, Chongqing 400044, China
}

Received July 22, 2010; accepted October 12, 2010

\begin{abstract}
$o$-hydroxyphenyl-triazine derivatives with formyl substituents were surveyed for the excited state intramolecular proton transfer (ESIPT). The occurrence of ESIPT was confirmed by well-separated emission bands for the derivatives. A low energy change from enol to keto in the excited state explains ESIPT for the derivatives.
\end{abstract}

excited state intramolecular proton transfer, $o$-hydroxyphenyl-triazine derivative, dual fluorescence

Citation: $\quad$ Li H L, Li H R, Yang L, et al. Excited state intramolecular proton transfer fluorescence emission of $o$-hydroxyphenyl-triazine derivatives. Chinese Sci Bull, 2011, 56: 1457-1460, doi: 10.1007/s11434-011-4473-4

Excited state intramolecular proton transfer (ESIPT) is a very important reaction in chemical and biological systems. It has received considerable interest in the areas of photochemistry, photophysics and photobiology since the investigation of the fluorescence of methyl salicylate [1,2]. Generally, ESIPT is achieved by an ultrafast photoinduced enol-keto tautomerization process during a four-level reaction $\left(\mathrm{E} \rightarrow \mathrm{E}^{*} \rightarrow \mathrm{K}^{*} \rightarrow \mathrm{K}, \mathrm{E}\right.$ : enol, $\mathrm{K}$ : keto) via five- [3,4], six[5], or rarely seven-membered quasi rings [6]. In this process, absorption occurs from $\mathrm{E} \rightarrow \mathrm{E}^{*}$ and emission from $\mathrm{K}^{*} \rightarrow \mathrm{K}$, which produces well-separated dual emission bands and an abnormally large stokes shift without self-absorption in the second emission band. $o$-hydroxyphenyl-triazine derivatives are well-known ultraviolet absorbers, that ESIPT is generally thought to occur in despite the lack of solid experimental evidence. Recent theoretical and experimental investigations of $o$-hydroxyphenyl-triazine derivatives have drawn opposite conclusions on this for the occurrence or absence of ESIPT, respectively [7-10]. Consequently, new $o$-hydroxyphenyl-triazine derivatives need to be developed and their spectral data analyzed to determine if ESIPT can occur in these compounds. In general, the reported $o$-hydro-

*Corresponding authors (email: hongruli1972@gmail.com; fanggao1971@gmail.com) xyphenyl-triazine derivatives have alkyl or aryl substituents on the hydroxyphenyl ring [7-10]. The electron-donating nature of alkyl or aryl groups could reduce the acidity of the hydroxy group and lower the possibility of ESIPT occurrence. In contrast, the acidity of the hydroxy group could be enhanced by electron withdrawing group, which would facilitate ESIPT. We recently investigated the occurrence of ESIPT in novel $o$-hydroxyphenyl- triazine derivatives (Figure 1, C1: 2-(2-hydroxy-5-formal- dehyde)-2H-benzotriazole, C2: 2-(2-methoxy-5-formalde- hyde)-2H-benzotriazole), which contained a formyl substituent on the hydroxyphenyl ring. To our knowledge, this is the first direct observation of the ESIPT emission of $o$-hydroxyphenyl-triazine derivatives.<smiles>O=Cc1ccc(O)c(-n2nc3ccccc3n2)c1</smiles>

C1<smiles>COc1ccc(C=O)cc1-n1nc2ccccc2n1</smiles>

C2
Figure 1 Chemical structures of the $o$-hydroxyphenyl-triazine derivatives. 
The derivatives $\mathbf{C} \mathbf{1}$ and $\mathbf{C 2}$ were synthesized by an established route (see Appendix). The final products were purified by column chromatography and the structures were characterized by ${ }^{1} \mathrm{H}-\mathrm{NMR}$ and elemental analysis (see Appendix). Figure 2 depicts the actual and normalized absorption spectra of $\mathbf{C 1}$ and $\mathbf{C 2}$ in cyclohexane, ethyl acetate, and acetonitrile from 260-400 nm. In these spectra, C1 displayed double absorption bands, while $\mathbf{C} 2$ exhibited a single absorption band. The maximum absorption wavelength of the first absorption band of $\mathbf{C 1}$ was similar to that of the absorption spectroscopy of C2 (Figure 2, Table 1). The second absorption band of $\mathbf{C 2}$ was red-shifted by approximately $60 \mathrm{~nm}$ with respect to the first absorption band (Table 1).

The data in Table 1 suggest clearly that the maximal absorption wavelength of first absorption band $\mathbf{C 1}$ is almost the same as the maximal absorption wavelength of $\mathbf{C 2}$, and the second absorption wavelength of $\mathbf{C 1}$ exhibits an approximately $60 \mathrm{~nm}$ red-shift with respect to them in various solvents. Both $\mathbf{C 1}$ absorption bands were observed in various solvents, including the aprotic and protic solvents cyclohexane, ethyl acetate, tetrahydrofuran, and methanol (Table 1). In contrast, $\mathbf{C 2}$ had only one absorption band in all the solvents. These results indicate that the longwavelength absorption band of $\mathbf{C} \mathbf{1}$ is not greatly affected by the polarity or protic nature of solvent. Furthermore, the double absorption bands of $\mathbf{C 1}$ exhibited almost no change on the addition of triethylamine or acetic acid to the solvents (see Appendix) while maintaining the concentration of C1. Even if in pure acetic acid or triethylamine, C1 still exhibits obvious long-wavelength absorption (see Appendix). Therefore the long-wavelength absorption of $\mathbf{C 1}$ probably does not arise from internal proton transfer, but it could be ascribed to the intramolecular hydrogen bond at the ground state.

Figure 3 shows the actual and normalized fluorescence spectra from 320-600 nm of $\mathbf{C 1}$ and $\mathbf{C 2}$ in cyclohexane, ethyl acetate and acetonitrile. Interestingly, C1 displayed well-separated double emission bands in ethyl acetate and acetonitrile, but only one emission band in cyclohexane. In
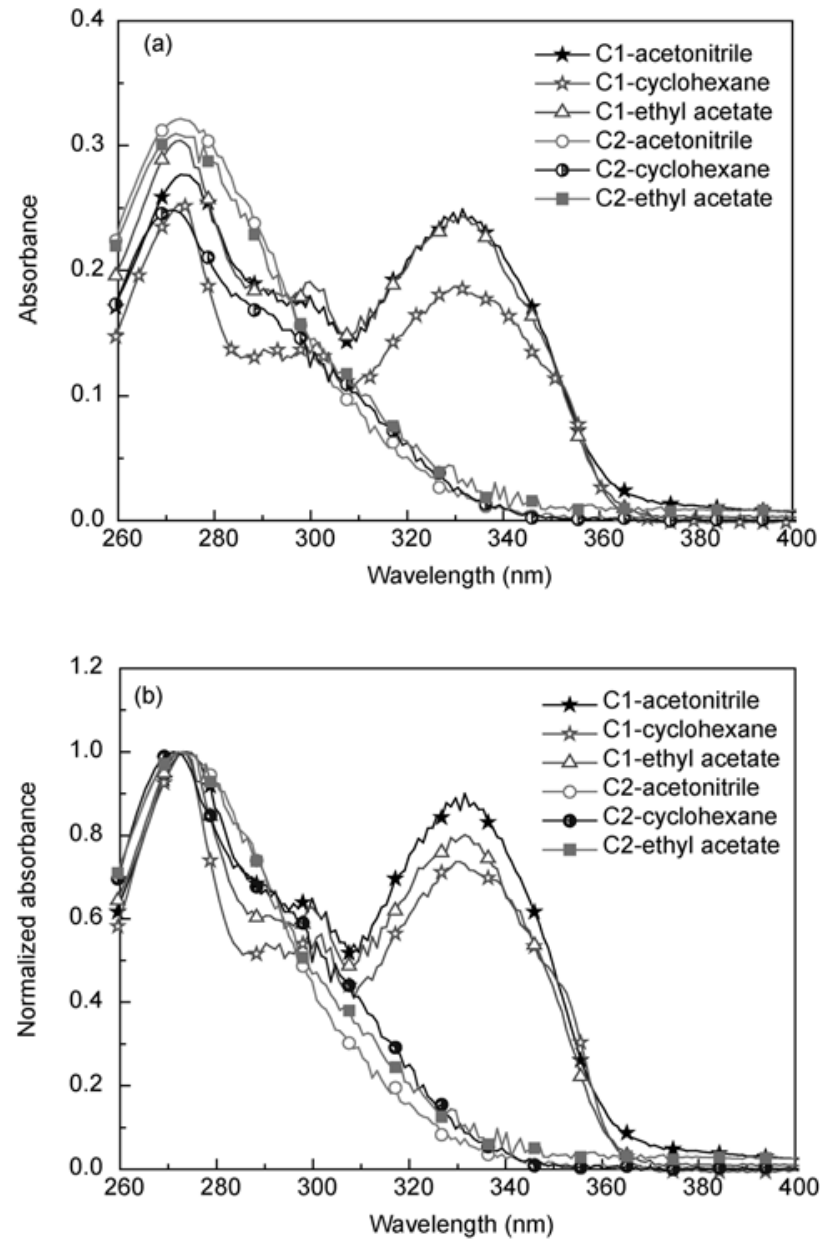

Figure 2 Ultraviolet/visible spectra of $\mathbf{C 1}$ and $\mathbf{C 2}$ in various solvents. (a) Actual spectra; (b) normalized spectra (C1, C2: $\left.1 \times 10^{-5} \mathrm{~mol} / \mathrm{L}\right)$.

sharp contrast, $\mathbf{C 2}$ displayed a single emission band in all solvents. The maximum emission wavelength of the first emission band of $\mathbf{C 1}$ was similar in ethyl acetate, acetonitrile and cyclohexane. Compared the first emission band, the second emission band of $\mathbf{C 1}$ had a much longer maximum emission wavelength in ethyl acetate and acetonitrile. This second emission band could be quenched by the

Table 1 Spectral parameters of $\mathbf{C 1}$ and $\mathbf{C 2}$ in various solvents ${ }^{\mathrm{a})}$

\begin{tabular}{|c|c|c|c|c|c|c|c|c|}
\hline \multirow{2}{*}{ Solvents } & \multicolumn{4}{|c|}{ C1 } & \multicolumn{4}{|c|}{$\mathrm{C2}$} \\
\hline & $\lambda_{\mathrm{a}, \max }$ & $\lambda_{\mathrm{f}, \text { max }}$ & $\Phi$ & $\varepsilon\left(\times 10^{-5}\right)$ & $\lambda_{\mathrm{a}, \max }$ & $\lambda_{\mathrm{f}, \max }$ & $\Phi$ & $\varepsilon\left(\times 10^{-5}\right)$ \\
\hline Cyclohexane & $\begin{array}{l}272 \\
329\end{array}$ & 345 & 0.068 & $\begin{array}{l}2.53 \\
1.87\end{array}$ & 270 & 368 & 0.053 & 2.49 \\
\hline Ethyl acetate & $\begin{array}{l}272 \\
330\end{array}$ & $\begin{array}{l}350 \\
489\end{array}$ & 0.016 & $\begin{array}{l}3.04 \\
2.43\end{array}$ & 271 & 385 & 0.088 & 3.09 \\
\hline Tetrahydrofuran & $\begin{array}{l}272 \\
329\end{array}$ & $\begin{array}{l}349 \\
479\end{array}$ & 0.011 & $\begin{array}{l}2.58 \\
2.01\end{array}$ & 273 & 380 & 0.063 & 3.32 \\
\hline Acetonitrile & $\begin{array}{l}272 \\
330\end{array}$ & $\begin{array}{l}357 \\
506\end{array}$ & 0.012 & $\begin{array}{l}2.77 \\
2.49\end{array}$ & 271 & 391 & 0.083 & 2.90 \\
\hline Methanol & $\begin{array}{l}279 \\
326\end{array}$ & 358 & 0.013 & $\begin{array}{l}2.15 \\
2.61\end{array}$ & 273 & 391 & 0.086 & 3.72 \\
\hline
\end{tabular}

a) $c(\mathbf{C 1})=1 \times 10^{-5} \mathrm{~mol} / \mathrm{L}, c(\mathbf{C 2})=1 \times 10^{-5} \mathrm{~mol} / \mathrm{L} . \lambda_{\mathrm{a}, \max }(\mathrm{nm})$, the maximum absorption wavelength; $\lambda_{\mathrm{f}, \max }(\mathrm{nm})$, the maximum emission wavelength. $\varepsilon$ (mol ${ }^{-1}$ $\left.\mathrm{cm}^{-1}\right)$, molar extinction coefficient. 

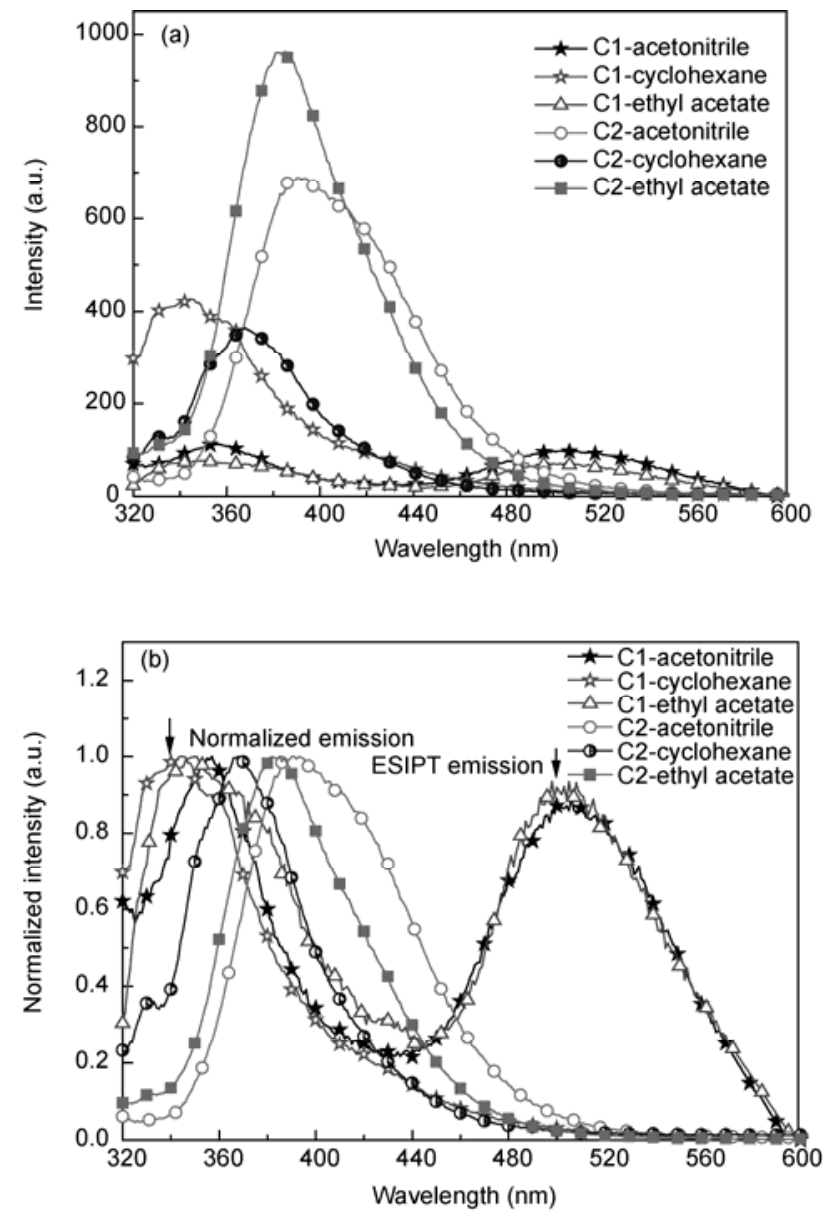

Figure 3 Fluorescence spectra of $\mathbf{C 1}$ and $\mathbf{C 2}$ in various solvents. (a) Actual spectra; (b) normalized spectra. Concentration of both $\mathbf{C 1}$ and $\mathbf{C 2}$, $1 \times 10^{-5} \mathrm{~mol} \mathrm{~L}^{-1}$, at the same excitation conditions.

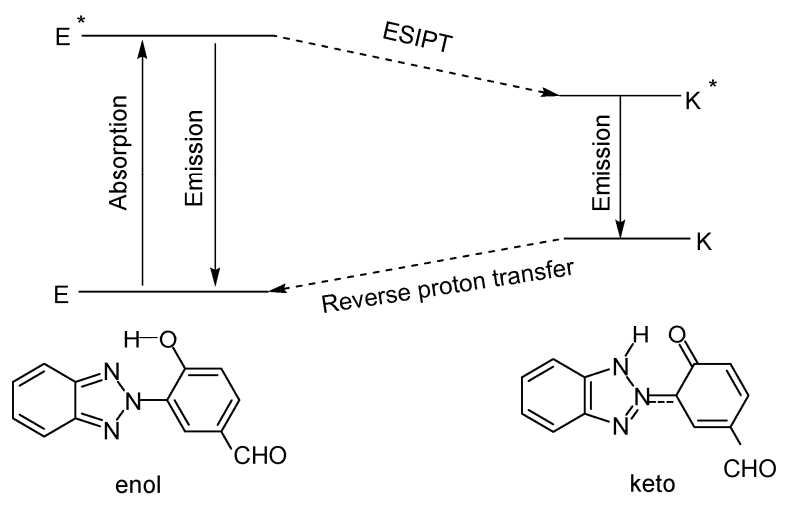

Figure 4 Four-level process of the excited state intramolecular proton transfer (ESIPT) of C1.

addition of methanol to the ethyl acetate or acetonitrile while maintaining the concentration of $\mathbf{C 1}$ (see Appendix). Consequently, it is reasonable that the second emission of C1 disappeared completely in methanol (see Appendix). The long-wavelength emission of $\mathbf{C 1}$ could also be reduced by adding acetic acid and triethylamine while maintaining the concentration of $\mathbf{C 1}$ (see Appendix). Further addition of acetic acid or triethylamine while maintaining the concentration of $\mathbf{C 1}$ resulted in recovery of the second emission band of $\mathbf{C 1}$ (see Appendix). The long-wavelength emission of $\mathbf{C 1}$ could not be detected in pure acetic acid or triethylamine (see Appendix). The second emission band of $\mathbf{C 1}$ exhibited an abnormally large Stokes shift of approximately $220 \mathrm{~nm}$ (Table 1). These results demonstrate that while the first emission band of $\mathbf{C 1}$ is from normal emission, the second emission band can be attributed to ESIPT. This means that the four-level reaction $\left(\mathrm{E} \rightarrow \mathrm{E}^{*} \rightarrow \mathrm{K}^{*} \rightarrow \mathrm{K}\right)$ via a six-membered quasi ring could take place in ethyl acetate and acetonitrile when C1 is excited (Figure 4). We also observed ESIPT emission of $\mathbf{C 1}$ in other strong aprotic polar solvents, such as DMF and DMSO (see Appendix). ESIPT emission of $\mathbf{C 1}$ in aprotic polar solvents could be suppressed by the addition of non-polar solvents, such as cyclohexane and benzene. On incremental addition of cyclohexane, the long- wavelength emission of $\mathbf{C 1}$ in ethyl acetate is gradually reduced and eventually completely disappeared (Figure 5). This indicates that the long-wavelength emission band of $\mathbf{C 1}$ is closely related to the polarity, acidity/basicity, and protic nature of the solvents, which is quite different to the long-wavelength absorption band. This observation is reasonable because the long-wavelength emission band of $\mathbf{C 1}$ is produced from an internal proton transfer process. The emission intensity of $\mathbf{C 1}$ was lower than that of $\mathbf{C 2}$ in all solvents except for cyclohexane under the same excitation conditions (Figure 3). As a result, the fluorescence emission quantum yields of $\mathbf{C 1}$ are much lower than those of $\mathbf{C 2}$ in all solvents except for cyclohexane (Table 1). It is accepted generally that fluorescence quantum yields of organic compounds are diminished by ESIPT. This means that the enol-keto tautomerization in the excited state weakens the emission of $\mathbf{C 1}$.

To understand the occurrence of ESIPT in C1, the energy changes from $\mathrm{E}^{*} \rightarrow \mathrm{K}^{*}$ for internal proton transfer were calculated for the $S_{0}$ and $S_{1}$ states (see Appendix), and plot-

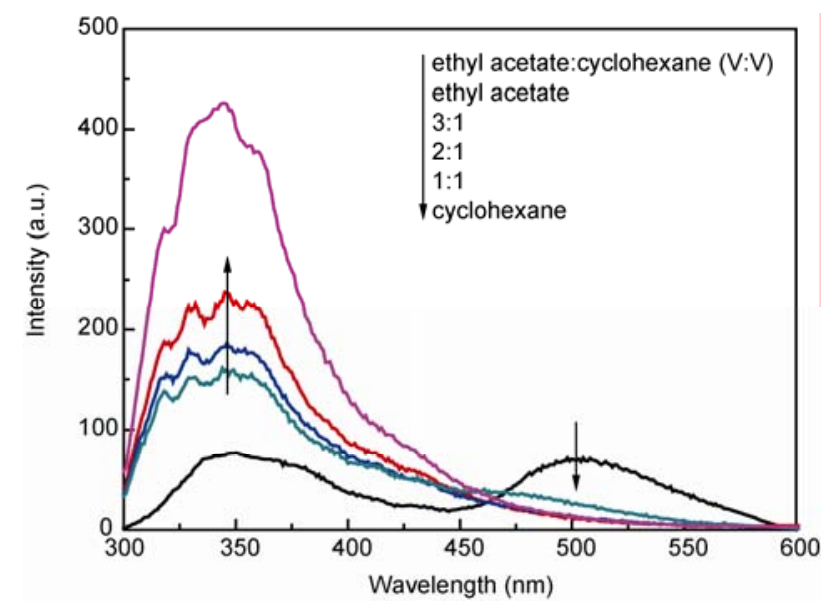

Figure 5 Fluorescence spectra of $\mathbf{C 1}$ in various binary ethyl acetate/cyclohexane solvents. The concentration of $\mathbf{C 1}$ was $1 \times 10^{-5} \mathrm{~mol} / \mathrm{L}$. 
ted as a function of $\mathrm{O}-\mathrm{H}$ distance (Figure 6). These energy changes will determine if ESIPT occurs, because it is a real chemical reaction. The energy plot (Figure 6) clearly shows that $\mathrm{E}^{*}$ is more stable than $\mathrm{K}^{*}$, and $\mathrm{K}$ is much more unstable than E. A small energy barrier $(16 \mathrm{~kJ} / \mathrm{mol})$ existed for the internal proton transfer from $E^{*} \rightarrow K^{*}$, which means molecular thermal motion is sufficient to overcome this barrier at room temperature. In contrast, the energy keeps increasing with $\mathrm{O}-\mathrm{H}$ distance from $\mathrm{E} \rightarrow \mathrm{K}$. This indicates that it is difficult for ground state intramolecular proton transfer $(\mathrm{E} \rightarrow \mathrm{K})$ to occur, while the reverse proton transfer process $(\mathrm{K} \rightarrow \mathrm{E})$ can take place easily. This is favorable for the occurrence of ESIPT. The calculations also confirmed that a large dipole moment $\left(4.19 \mathrm{D}, 1 \mathrm{D}=6.695 \times 10^{-30} \mathrm{C} \mathrm{m}\right)$ change occurs for $\mathrm{E}^{*} \rightarrow \mathrm{K}^{*}$. This in turn explains why the solvent polarity is

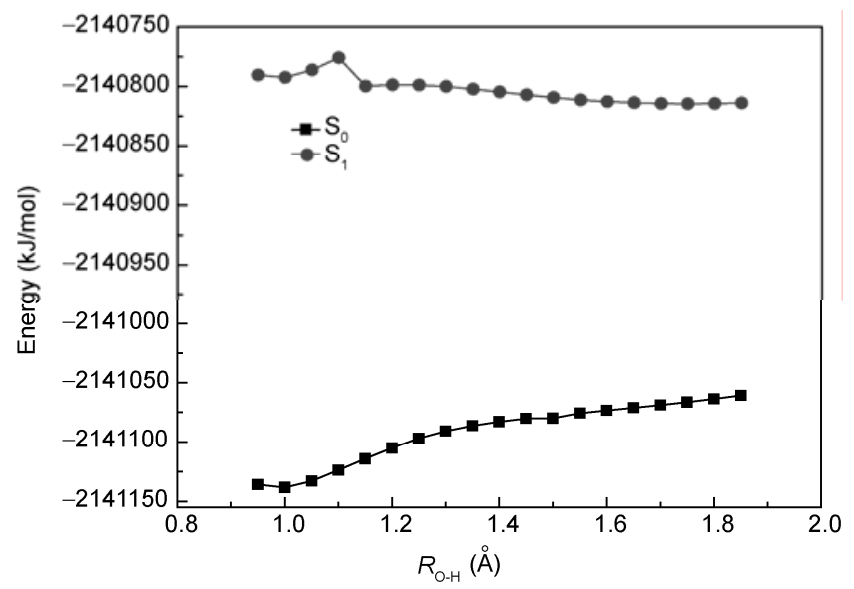

Figure 6 Plots of energy as a function of $\mathrm{O}-\mathrm{H}$ distance for enol to keto tautomerization in the $\mathrm{S}_{0}$ and $\mathrm{S}_{1}$ states of $\mathbf{C 1}$. favorable for ESIPT occurrence, as observed in our experiments. This is mainly due to the occurrence of a huge structural reorganization for $\mathbf{C} 1$ during ESIPT.

In summary, these results illustrate the satisfactory wellseparated ESIPT emission bands for a novel $o$-hydroxyphenyl-triazine derivative. This provides strong experimental and theoretical evidence that $o$-hydroxyphenyl-triazine derivatives are able to undergo ESIPT. In consideration of its structural simplicity and modifiability, $\mathbf{C 1}$ could be an ideal model for the development of novel $o$-hydroxyphenyl-triazine derivatives that can undergo ESIPT. These derivatives could also be used to probe the ESIPT dynamics of $o$-hydroxyphenyl-triazine systems.

This work was supported by the Fundamental Research Funds for the Central Universities (CDJZR10220006). We appreciate warm supports from the Key Laboratory of Functional Crystals and Laser Technology, TIPC, Chinese Academy of Sciences.

1 Weller A. Z Elektrochem, 1956, 60: 1144-1147

2 Mitra S, Mukherjee S. J Lumine, 2006, 118: 1-11

3 Yu W S, Cheng C C, Cheng Y P, et al. J Am Chem Soc, 2003, 125: 10800-10801

4 Chen C L, Lin C W, Hsieh C C, et al. J Phys Chem A, 2009, 113: 205-214

5 Chen K Y, Hsieh C C, Cheng Y M, et al. Chem Commun, 2006, 4395-4397

6 Chen K Y, Cheng Y M, Lai C H, et al. J Am Chem Soc, 2007, 129: 4534-4535

7 Paterson M J, Robb M A, Blancafort L, et al. J Phys Chem A, 2005, 109: 7527-7537

8 Fluegge A P, Waiblinger F, Stein M, et al. J Phys Chem A, 2007, 111: 9733-9744

9 Paterson M J, Robb M A, Blancafort L, et al. J Am Chem Soc, 2004, 126: $2912-2922$

10 Maliakal A, Lem G, Turro N, et al. J Phys Chem A, 2002, 106: 7680-7689

Open Access This article is distributed under the terms of the Creative Commons Attribution License which permits any use, distribution, and reproduction in any medium, provided the original author(s) and source are credited.

\section{Supporting Information}

Appendix Synthesis of $\mathbf{C 1}$ and $\mathbf{C 2}$, theroretical calculations and measurement of spectral properties

The supporting information is available online at csb.scichina.com and www.springerlink.com. The supporting materials are published as submitted, without typesetting or editing. The responsibility for scientific accuracy and content remains entirely with the authors. 